\title{
Mathematical Model for Thermal Tracking and On-line Control in Continuous Casting
}

\author{
K.-H. SPITZER, K. HARSTE, ${ }^{1)}$ B. WEBER, ${ }^{2)}$ P. MONHEIM ${ }^{3)}$ and K. SCHWERDTFEGER
}

Institut für Allgemeine Metallurgie, Technische Universität Clausthal, Robert-Koch-Str. 42, D-3392 Clausthal-Zellerfeld, Germany. 1) Dillinger-Hüttenwerke, Postfach 1580, D-6638 Dillingen/Saar, Germany. 2) Mannesmann Datenverarbeitung, Rehhecke 50, D-4030 Ratingen 4, Germany. 3) Mannesmann Demag Hüttentechnik, Wolfgang-ReuterPlatz, D-4100 Duisburg 1, Germany.

(Received on January 6, 1992; accepted in final form on April 24, 1992)

\begin{abstract}
A mathematical model has been developed to calculate the three dimensional and instationary temperature field in the strand during continuous casting. The numerical algorithm and the computer program were tested by application to the Neumann problem for which an analytical solution is available. It was found that this analytical solution is reproduced quite closely even if relatively coarse finite difference meshes are used. An algorithm based on the solution of the inverse heat conduction problem is applied to adjust the heat transfer coefficients for the computer model using the results of surface temperature measurements. Another algorithm was developed to calculate the material data from the steel composition. This algorithm is applicable to steel grades with low contents of alloying elements. The model fulfills the requirements concerning computing time consumption and accuracy for an on-line application. Results obtained with the model which is installed at several casters are presented.
\end{abstract}

KEY WORDS: continuous casting; on-line control; heat transfer model; inverse model; mathematical modeling.

\section{Introduction}

In the continuous casting of steel computer-aided process control and mathematical modeling are important tools for the improvement of product quality and productivity. A process control system ${ }^{1-3)}$ has to ensure that the different casting parameters like the amount of spray water and the casting speed fit together. A too strong or too weak cooling can cause cracks or bulging and, consequently, reduces the product quality. Controlling the amount of spray water in the various zones depending on the strand history is an effective counter measure for such problems. ${ }^{4}$ The rules for calculating the correct amount of spray water are derived both from empirical data like measured surface temperatures and by mathematical modeling. A fundamental type of modeling is the simulation of the solidification process. ${ }^{4-11)}$ Such a simulation gives directly important data like surface temperatures and shell thickness. By inclusion of a segregation model concentration profiles $^{11.12)}$ can be calculated and, by using additional empirical relations, the solidification structure can be predicted. $^{13)}$

In most solidification models either a one-dimensional $^{4,5,9,10)}$ line or a two-dimensional ${ }^{6,7,8)}$ cross-sectional area of the strand is followed on its way through the caster. The equation for heat transport is solved numerically, mostly by applying an explicit finite difference method. Finite elements ${ }^{8)}$ are relatively seldom used. Implicit finite difference methods, although offering numerous advantages over explicit methods, are likewise unpopular for the modeling of continuous casting, probably because they lead to more complicated algorithms. Reviews on the numerous methods to solve the heat transport equation numerically can be found in the literature. ${ }^{14,15)}$

Usually, the mathematical modeling of continuous casting is used to improve the basic understanding and by this to provide information for caster design and operation. The on-line use of numerical solidification models for direct process control, ${ }^{4,16)}$ which is the main aim of the work reported in this paper, has not been applied frequently.

The developed on-line model is used to calculate the instationary temperature field in a longitudinal crosssection through the middle of the slab. Special requirements concerning computing time and accuracy are to be fulfilled and special process conditions, such as the start of casting, must be taken into account. In order to meet such requirements implicit enthalpy methods are the most appropriate. For off-line simulations, an extended model calculating the instationary temperature distribution in the full threedimensional strand was developed which is used, for instance, for caster design or optimization of secondary cooling.

For the simulation of solidification in continuous casting the knowledge of the correct boundary conditions 
is crucial. Accuracy requirements for process control application make surface temperature measurements necessary. The results of such measurements are applied to adjust the mathematical boundary conditions by using an algorithm based on the solution of the inverse heat transport problem.

The temperature dependent thermal conductivity, density and enthalpy which are the material data of the steel necessary for the model are calculated from the chemical composition. The computer program written for this purpose is applicable to low alloyed steels.

\section{Mathematical Model}

The temperature field in the strand is described by the heat transport equation. Assuming that

- increased heat transport in the liquid core due to convection can be taken into account by an increased effective thermal conductivity $\left(\lambda=\lambda_{\text {eff }}\right)$,

- the movement of the shell due to shrinkage is small compared to the casting speed $\left(v_{z}=v_{\text {cast }}\right)$, and

- heat conduction in the casting direction is small compared to convective transport due to casting speed $\left(|(\partial / \partial z)(\lambda \cdot \partial T / \partial z)| \ll\left|\rho v_{\text {cast }} \cdot \partial H / \partial z\right|\right)$.

This equation takes the form (symbols are explained separately.)

$$
\rho\left(\frac{\partial H}{\partial t}+v_{\text {cast }} \frac{\partial H}{\partial z}\right)=\frac{\partial}{\partial x}\left(\lambda \frac{\partial T}{\partial x}\right)+\frac{\partial}{\partial y}\left(\lambda \frac{\partial T}{\partial y}\right) .
$$

During the numerical solution of Eq. (1) problems can arise if $\lambda$ depends strongly on $T$, for instance, if $\lambda\left(T_{l}\right)$ is made several times larger than $\lambda\left(T_{s}\right)$. In this case $|(\partial \lambda / \partial x)(\partial T / \partial x)| \gg\left|\lambda \cdot \partial^{2} T / \partial x^{2}\right|$ or $|(\partial \lambda / \partial y)(\partial T / \partial y)| \gg$ $\left|\lambda \cdot \partial^{2} T / \partial y^{2}\right|$ might be valid in the temperature range between $T_{s}$ and $T_{l}$ not allowing to use central differences for $\partial T / \partial x$ or $\partial T / \partial y$ in the numerical calculation. A well known method to avoid such problems is the use of a transformed temperature (Kirchoff transformation)

$$
\tilde{T}=\int_{T_{o}}^{T} \lambda(T) d T
$$

where $T_{0}$ is some fixed but arbitrary reference temperature. Using this transformed temperature Eq. (1) takes the form

$$
\rho\left(\frac{\partial H}{\partial t}+v_{\text {cast }} \frac{\partial H}{\partial z}\right)=\frac{\partial^{2} \widetilde{T}}{\partial x^{2}}+\frac{\partial^{2} \tilde{T}}{\partial y^{2}}
$$

If the temperature is calculated only in a longitudinal cross-sectional area through the middle of the slab where temperature gradients in $y$-direction can be neglected, Eq. (1-a) simplifies to

$$
\rho\left(\frac{\partial H}{\partial t}+v_{\text {cast }} \frac{\partial H}{\partial z}\right)=\frac{\partial^{2} \tilde{T}}{\partial x^{2}}
$$

It is assumed that $H, \rho$ and $\lambda$ are only functions of $T$ or $\tilde{T}$ respectively (equilibrium solidification) and of the steel composition.

During the regular casting time Eq. (1-a) is solved in a domain bounded from above by the meniscus $(z=0)$ and ending at $z=z_{o}$, Fig. 1. At the start of casting $z_{o}$ is

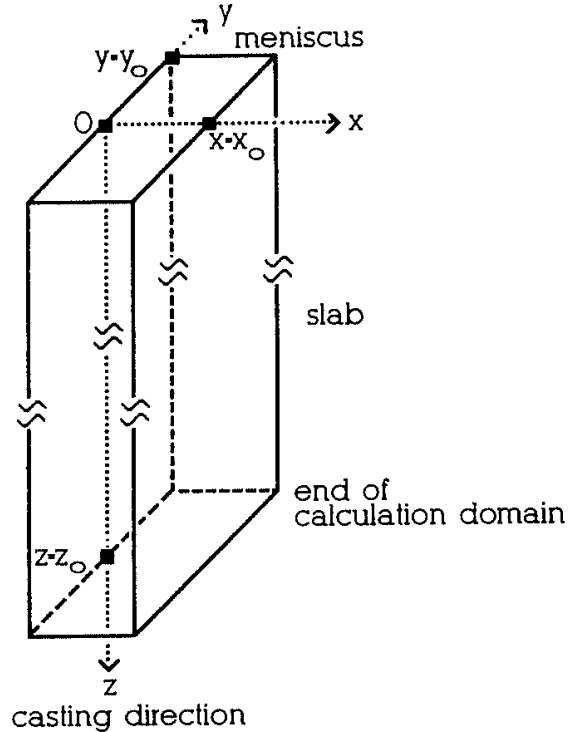

Fig. 1. Coordinate system used for the calculations.

the actual position of the strand end (position of the dummy bar head). Correspondingly, at the end of casting the upper boundary of the calculation domain, defined by the meniscus or strand top, moves downwards with the withdrawal speed of the strand. So the calculation starts with some $z_{\mathrm{top}}>0$. After a change in steel composition, the boundary between the two steel grades is followed on its way through the caster, and in the corresponding strand sections different material data can be used. Initial and boundary conditions are specified as follows:

$t=0,0 \leq z \leq z_{0}: \quad T=T_{\text {cast }}$

$z=0$ :

strand surface: $\quad-\lambda \frac{\partial T}{\partial n}=-\frac{\partial \tilde{T}}{\partial n}=q$

with

$$
q=h\left(T_{\text {surface }}-T_{\text {surrounding }}\right)+\sigma \varepsilon T_{\text {surface }}^{4}
$$

plane of symmetry:

$$
q=0
$$

A plane of symmetry is assumed if the calculation is performed for only one half of the slab. In this case the plane of symmetry may be located either at $x=0$ or at $x=x_{o}$.

The last term in Eq. (6-a) which is omitted in the mould area presents the heat transfer by radiation. The casting temperature $T_{\text {cast }}$ is close to the tundish temperature which is measured frequently and is calculated from the latter by an empirical relation. For $T_{\text {surrounding }}$ in the mould and in the spray zones the water temperature is used.

The heat transfer coefficient $h$ is a local function on the slab surface. In all cooling zones including the mould and the radiation area an expression of the form

$$
h_{i}=a_{i} \xi^{2}+b_{i} \xi+c_{i}
$$

is used. $\xi$ is a variable which can present different types of process parameters depending whether it is for the 
mould or a spray cooling zone. The index $i$ is the current number of the zone. As will be explained later, the parameters $a_{i}, b_{i}, c_{i}$ are determined to give optimum agreement to measured surface temperatures. Other types of functions, e.g. $h_{i}=a_{i} \xi^{b_{i}}+c_{i}$ were tested but the obtained agreement was not as good as with Eq. (7).

In the mould region the casting speed can be used for $\xi$. To take account for gap formation in the mould due to strand shrinkage Eq. (7) can be multiplied additionally by a $z$-dependent term, for instance by a linear function decreasing from one with increasing distance from the meniscus.

If mould cooling water temperatures and water rates are measured continuously, the averaged heat flux density in the mould can be directly calculated. This averaged heat flux density, or a heat flux density distribution giving the same average value, can be inserted for $q$ in Eq. (5) instead of using a heat transfer coefficient $h$ according to Eq. (7) in Eq. (6-a). If this is done the effect of steel grade, ${ }^{17)}$ of casting flux and of casting velocity is taken directly into account. But when following this procedure the reliability of the measurements for the mould cooling water rate and temperature increase must be carefully considered. A mistake in the heat flux density has a significantly stronger effect on the calculated results than a mistake of the same percentage for the heat transfer coefficient. Consequently, a heat transfer coefficient as a function of casting speed with proper chosen parameters in Eq. (7) might often yield the better results.

In the secondary cooling zones $\xi$ is the spray water density. After the end of the spray area a heat transfer coefficient with $a_{i}=b_{i}=0$ is used accounting for the effect of thermal convection. The parameter $c_{i}(=h)$ may still depend on the position on the strand, if this area is subdivided into several areas.

If local variations in the temperature distribution are of interest, a more detailed spatial distribution of $h$ can be used to take the effect of roll contact and non-uniform spray water density etc. into account. Of course, it is then necessary that corresponding data, from detailed measurements, are available to determine the empirical parameters $a_{i}, b_{i}, c_{i}$ in Eq. (7).

These empirical parameters will vary within certain limits for each individual caster. An initial estimate for the parameters can be taken from the literature. ${ }^{5-10,17-20)}$ The procedure to adjust the coefficients to the specific conditions on the basis of surface temperature measurements will be explained later.

During the start of casting the length of the calculation domain increases. So, for a grid point $(z, t)$ of the finite difference mesh with $z_{o}(t-\Delta t)<z<z_{o}(t)$ where $\Delta t$ is the time increment in the discretization, there is no value $H(z, t-\Delta t)$ available to derive the finite difference expression for $\partial H / \partial t$ directly. Such an expression can be easily obtained together with that for $\partial H / \partial z$ by a Taylor series expansion at the point $(z, t)$ for $H\left(z_{o}(t-\Delta t), t-\Delta t\right)$ and $H(z-\Delta z, t)$ where $\Delta z$ is the mesh width in $z$-direction.

At the end of casting, when the meniscus moves downwards $\left(z_{\text {top }} \geq 0\right)$ Eq. (4) can no longer be used as boundary condition. Then the meniscus area is regarded as a slice moving down with casting speed. The temperature in this slice can be calculated by Eq. (1-a) without the term $v_{\text {cast }} \cdot \partial H / \partial z$ if the coordinate system is fixed at the slice.

Of course, for the part of the strand in the vicinity of the dummy bar at the start of casting and for the part near the meniscus at the end of casting, the assumption that $\partial^{2} \widetilde{T} / \partial z^{2}$ (heat conduction in $z$-direction) can be neglected, is no longer justified. The inclusion of $\partial^{2} \tilde{T} / \partial z^{2}$ into Eq. (1-a) or (1-b) and the use of boundary conditions for the meniscus area and the contact area to the dummy bar, e.g. defined by some proper heat transfer coefficients, is in principle no problem. But even if the term $\partial^{2} \widetilde{T} / \partial z^{2}$ is considered only in these shorter parts of the strand, while Eq. (1-a) or (1-b) is used for the rest inbetween, the computing time requirements increase significantly. On the other hand the first and last one or two meters of the total cast length which correspond to the parts of the strand where heat conduction in $z$-direction cannot be neglected, are usually not of great practical interest. Therefore, this more complicated procedure for the treatment of the first and last part of the strand is not to be recommended.

For the on-line application of the model the following requirements for the numerical algorithm are to be fulfilled:

- the numerical algorithm must be fast enough for parallel real time simulation of several strands with sufficient accuracy using a process computer. Simultaneously this computer must also take over other jobs such as quality control or cut length optimization.

- during periods in which insufficient computer capacity is available for the program, it must be able to use increased time steps. Of course, this reduces the accuracy.

The enthalpy method combined with an implicit finite difference scheme is believed to be the most appropriate method to meet such requirements. A sketch of the developed algorithm is given in the Appendix.

\section{Test of the Model}

In order to test the model and to check the effect of numerical discretization on accuracy, the model was applied to the theoretical case of continuous casting of pure iron $\left(T_{l}=T_{s}\right)$ under steady state conditions. It is assumed that there is no superheat, that all the material data and the surface temperature $T_{\text {surface }}$ at one face $(x=0)$ are constant. With the boundary condition $q=0$ at the other face $\left(x=x_{0}\right)$ the temperature profile up to the point of final solidification is given by the well known Neumann solution. This solution can also be used after the end of solidification (the whole domain being solid) if the heat flux calculated from the derivation of Neumann's analytical formula (at $x=x_{o}$ ) is used as boundary condition. Since the numerical model is applied to predict temperatures using a heat transfer coefficient at the surface $(x=0)$, Neumann's solution is also used with a heat transfer coefficient rather than with a constant surface temperature condition and the check between the 


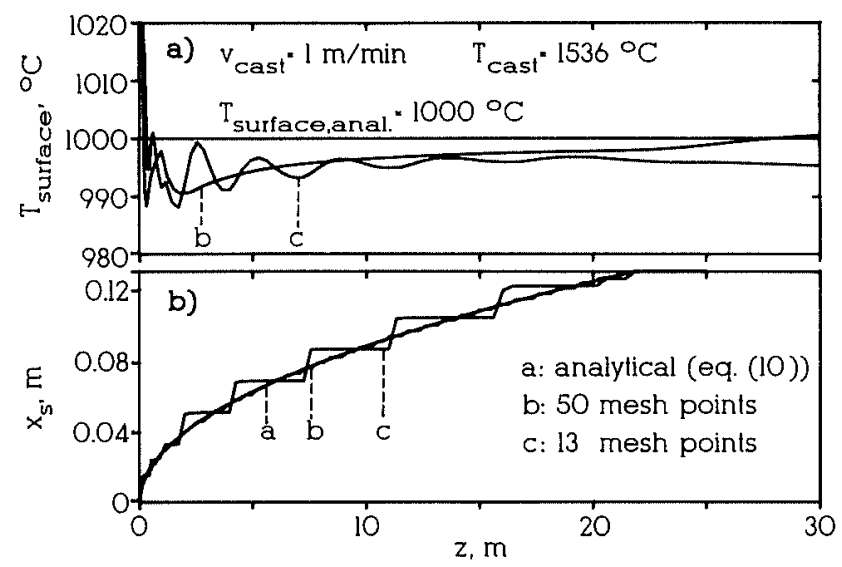

Fig. 2. Difference between calculated and analytical surface temperature (a), and shell thickness (b), respectively. The numerical calculations are performed with two different meshes.

Table 1. Material data of pure iron used in the test of the model.

\begin{tabular}{|c|c|}
\hline Thermal conductivity & $\lambda=33 \mathrm{~W} \mathrm{~m}^{-1} \mathrm{~K}^{-1}$ \\
\hline Density & $\rho=7400 \mathrm{~kg} \mathrm{~m}^{-3}$ \\
\hline Specific heat & $c_{p}=700 \mathrm{~J} \mathrm{~kg}^{-1} \mathrm{~K}^{-1}$ \\
\hline Latent heat & $\Delta H=260 \mathrm{~kJ} \mathrm{~kg}^{-1}$ \\
\hline Liquidus temperature & $: T_{l}=T_{s}=1536^{\circ} \mathrm{C}$ \\
\hline
\end{tabular}

numercial and analytical calculation will be in the surface temperature and the shell thickness. The heat transfer coefficient obtained from the analytical temperature profile is.

$$
\begin{aligned}
x=0: \quad h & =\frac{\lambda}{\left(T_{\text {surface }}-T_{\text {surrounding }}\right)} \frac{\partial T}{\partial x} \\
& =\frac{\lambda\left(T_{l}-T_{\text {surface }}\right)}{\left(T_{\text {surface }}-T_{\text {surrounding }}\right) \sqrt{\pi \kappa z / v_{\text {cast }}} \operatorname{erf} \eta}
\end{aligned}
$$

The parameter $\eta$ is given by

$$
\sqrt{\pi} \eta e^{\eta^{2}} \operatorname{erf} \eta=\frac{c_{p}\left(T_{l}-T_{\text {surface }}\right)}{\Delta H}
$$

A comparison of the numerical results of the model with the corresponding analytical expressions is shown in Fig. 2.

The numerical calculations were performed with two finite difference meshes, with 13 or 50 points respectively in $x$-direction and 160 points in $z$-direction. The material data of pure iron ${ }^{21)}$ are given in Table 1.

Figure 2a) shows the difference between the fixed analytical surface temperature of $1000^{\circ} \mathrm{C}$ and $T_{\text {surface }}$ resulting from the numerical calculation. As can be seen, apart from a small range near the meniscus where there is a discontinuous decrease from 1536 to $1000^{\circ} \mathrm{C}$ for the analytical solution, this difference is limited to a few degrees, even for the coarse mesh (13 points), which easily fulfills the computing time requirements for the on-line application.

Figure 2b) shows the thickness of the solidified shell for the analytical solution given by

$$
x_{s}=2 \eta \sqrt{\kappa z / v_{\text {cast }}}
$$

and for the numerical calculations. The numerically obtained shell thicknesses reveal a step form which is characteristic for the finite difference enthalpy method. As can be seen, this effect decreases with increasing number of mesh points in the $x$-direction. The curves fit to the step functions give a close approximation to the exact analytical solution even for the coarse 13-point mesh. Methods to prevent the jumping of the solidification front from one to the next grid point are known ${ }^{22)}$ but are unnecessary for the present purpose.

Summing up it can be said that the mathematical model is able to predict the "continuous casting of pure iron" with a very good accuracy even for a quite coarse grid. To obtain such an agreement a refinement of the mesh (decreasing distance between the mesh points) near the boundaries (surfaces or centre line) is of great importance. So it can be assumed that the model will also yield a good prediction for real continuous casting provided that the material data and the heat transfer coefficients used are correct.

\section{Determination of Heat Transfer Coefficients from Measurements}

The knowledge of the heat transfer rates at the strand surface which means that of the correct coefficients in formula (7) is a prerequisite for a successful application of the described model. As mentioned previously, a first estimate of the parameters in Eq. (7) can be derived from the literature data..$^{5-10,17-20)}$ For a specific caster these parameters can be adjusted more precisely based on surface temperature measurements, for instance, using an optical pyrometer.

The following procedure which is used off-line can be applied:

A strand slice is tracked on its way through the caster during the casting process. The strand slice starts its travel at the meniscus at the process time $t=t_{0}$. The time scale of the slice is $t^{\prime}$ (zero at $t=t_{o}$ ). Hence, the position $z$ of the slice during its travel is

$$
z\left(t^{\prime}\right)=\int_{t_{o}}^{t_{o}+t^{\prime}} v_{\text {cast }}(t) d t
$$

So it is known at which $z$ (mould or in one of the spray zones) the slice is located at each time and, therefore, from the recorded process data the corresponding spray water density or temperature increase of the mould water (value for $\xi$ in Eq. (7)) is obtained as well. The temperature profile in the slice can then be calculated by Eq. (1-b) using $t^{\prime}$ instead of $t$ and ommitting $v_{\text {cast }} \cdot \partial H / \partial z$, applying the initial and boundary conditions

$$
\begin{aligned}
& T\left(t^{\prime}=0\right)=T_{\text {cast }}\left(t=t_{o}\right) \\
& -\lambda \frac{\partial T}{\partial n}\left(t^{\prime}\right)=-\frac{\partial \tilde{T}}{\partial n}\left(t^{\prime}\right)=h\left(\xi(t), a\left(t^{\prime}\right), b\left(t^{\prime}\right), c\left(t^{\prime}\right)\right) \\
& \cdot\left(T_{\text {surface }}\left(t^{\prime}\right)-T_{\text {surrounding }}(t)\right)+\sigma \varepsilon T_{\text {surface }}^{4}\left(t^{\prime}\right)
\end{aligned}
$$

Since the process parameters $\xi, T_{\text {cast }}, T_{\text {surrounding, }}, v_{\text {cast }}$ depend on the manner of operating the caster, that is on the process time $t$, the calculation will yield dif- 
ferent results for slices with other process parameters.

If the surface temperatures are measured during casting at several positions $k$, then the temperature $T_{\text {surface }}\left(t_{k}^{\prime}\right)$ of the slice at the time $t_{k}^{\prime}$ when the slice is at the measuring position with current number $k$ can be compared to the corresponding measured temperature $T_{\text {measured, } k}\left(t_{o}+t_{k}^{\prime}\right)$. The problem to be solved is to find those parameters in Eq. (7) giving the optimum agreement between measurement and calculation. For this the following objective function is minimized.

$$
\begin{aligned}
& F\left(\left(a_{i}, b_{i}, c_{i}\right)_{\text {all used zones }}\right) \\
& =\frac{1}{n_{s}} \sum_{\text {slices }} \frac{1}{n_{k}} \sum_{k=1}^{n_{k}}\left(\frac{T_{\text {measured, } k}\left(t_{o}+t_{k}^{\prime}\right)-T_{\text {surface }}\left(t_{k}^{\prime}\right)}{T_{\text {measured }, k}\left(t_{o}+t_{k}^{\prime}\right)}\right)^{2}
\end{aligned}
$$

The LHS signifies that $F$ is a function of the parameters $a, b, c$. For instance, if there are 4 different cooling zones along the strand and for each zone all of the three parameters are used, $F$ will be a function of 12 parameters of which its minimum value is to be determined. The summation on the RHS is first over the $n_{k}$ measuring positions and then over $n_{\mathrm{s}}$ slices which were followed. The objective function gives a normalized (by division through the measured value) and averaged square difference between measured and calculated temperatures. Other terms like differences between measured and calculated heat fluxes in the mould can be easily included into Eq. (14). In this case without normalization the optimization procedure would tend to ignore temperature data, because the heat fluxes are (depending on the chosen system of units) several orders of magnitude larger. The set of strand slices used for the optimization should be chosen in such a manner that a large range of process parameters $\left(\xi, v_{\text {cast }}\right.$, steel grade etc.) is covered.

If only one pyrometer is available the method can be modified in such a way that measurements are performed subsequently at different positions. The optimum parameters are determined step by step (with $n_{k}=1$ in Eq. (14)) for the zones between two neighbouring measuring positions while the already optimized parameters for the zones located above are left unchanged.

To minimize the objective function Eq. (14) a more or less conventional gradient method is used. The algorithm was tested in such a way that first "true" coefficients in Eq. (7) were chosen and that temperatures were calculated for different process parameters. These temperatures were used as "measured" temperatures. Starting with wrong coefficients in Eq. (7), the objective function was minimized. The resulting new coefficients were sometimes found to differ significantly from the "true" ones but the functions for $h$ obtained when inserting both the new and the "true" coefficients into Eq. (7) were very close to each other in the range of $\xi$ covered by the set of slices.

Of course, the method described above cannot provide more information on the process than that contained in the measured data supplemented by the governing physical relations. The main advantage is that a huge amount of data can be analyzed automatically by the computer where a pure trial and error approach would be without hope. Similar optimization techniques as described have been used by other authors ${ }^{23,24)}$ to find optimum process strategies in continuous casting.

\section{Application of the Model}

The model has been installed at several casters. The subsequent results were obtained at the slab caster of the Dillinger Hüttenwerke (Dillingen, Germany). The caster has five secondary cooling zones. To adjust the parameters in the Eq. (7) for the heat transfer coefficients the caster was equipped with five pyrometers. In the mould the heat transfer coefficient was made a function of the casting speed. From different casts seven strand slices were selected covering different casting conditions and steel grades. So 18 parameters (mould plus five secondary cooling zones) are to be adjusted to minimize 35 temperature differences. Starting with a set of estimated parameters a first series of optimization runs was performed where only the $c_{i}$ 's in Eq. (7) were varied. After this all the parameters were included in the optimization. The effect reached by the adjustment of the parameters can be seen in Figs. 3-5. Figure 3 shows the surface temperatures as a function of the distance from the meniscus calculated both with the estimated and the adjusted parameters. The measured temperatures are given by the symbols. The results shown in this figure are for a typical steady state casting condition.

In Fig. $\mathbf{4}$ for the first pyrometer position (uppermost pyrometer) the calculated temperatures as a function of the time after start of casting are compared to the measured temperatures. Again both the results obtained with the estimated and with the adjusted parameters are shown. Within the presented period casting speed and spray water densities were varied.

The absolute value of the temperature difference between measurements and calculations averaged over all measuring points is given in Fig. 5 as a function of time. The absolute values must be used because averaged differences might be zero although the single differences are not. Summing up it can be said that the adjusted

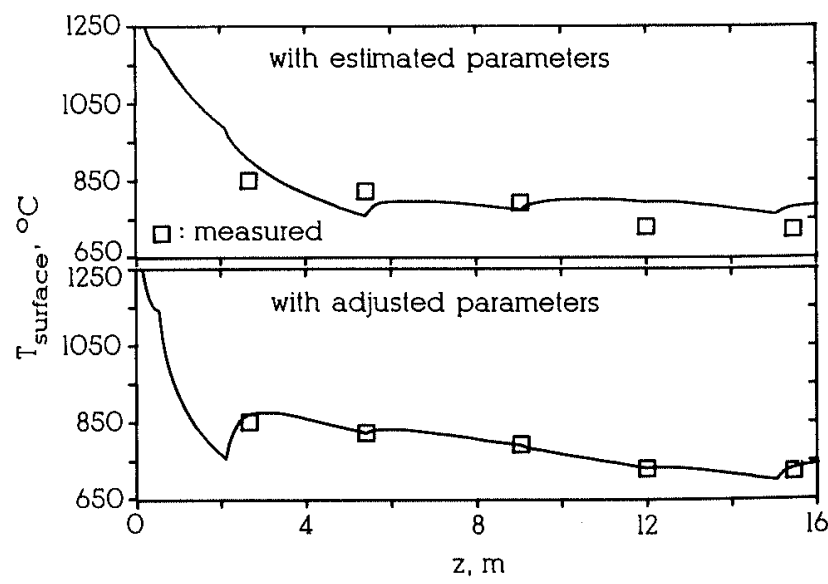

Fig. 3. Measured and calculated surface temperatures as a function of the distance form the meniscus obtained with the estimated and adjusted parameters (slab caster at Dillinger-Hüttenwerke). 


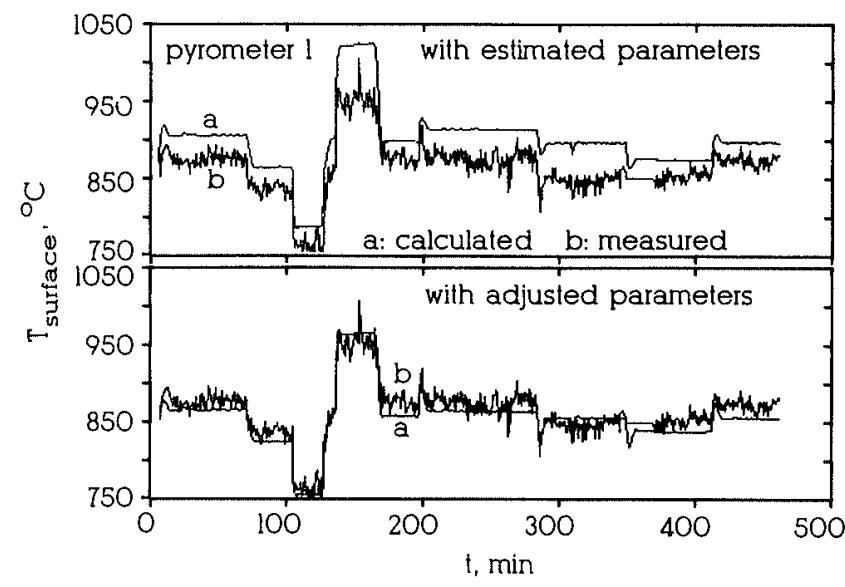

Fig. 4. Measured and calculated surface temperature as a function of time at the position of the first pyrometer.

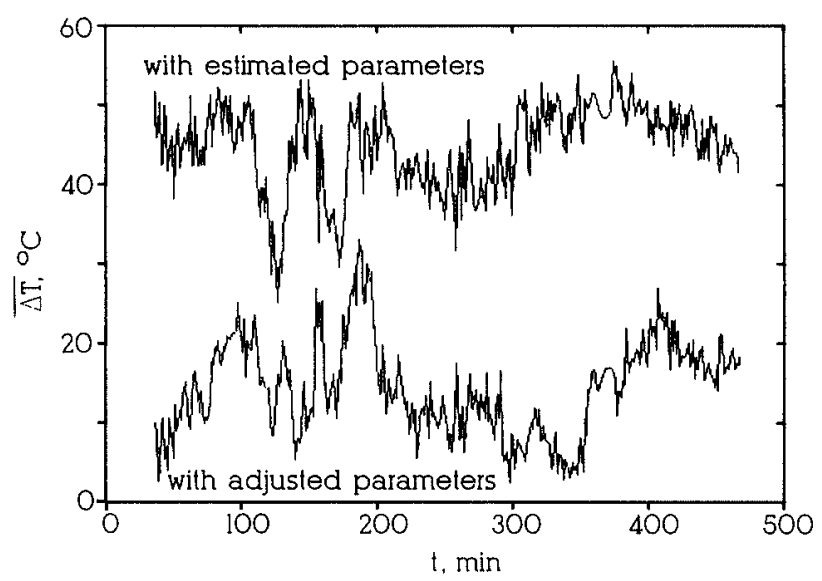

Fig. 5. Difference $\overline{\Delta T}=(1 / 5) \sum_{j=1}^{S}\left|T_{\text {measured, } j}-T_{\text {calculated, } j}\right|$ as a function of time obtained with the estimated and the adjusted parameters.

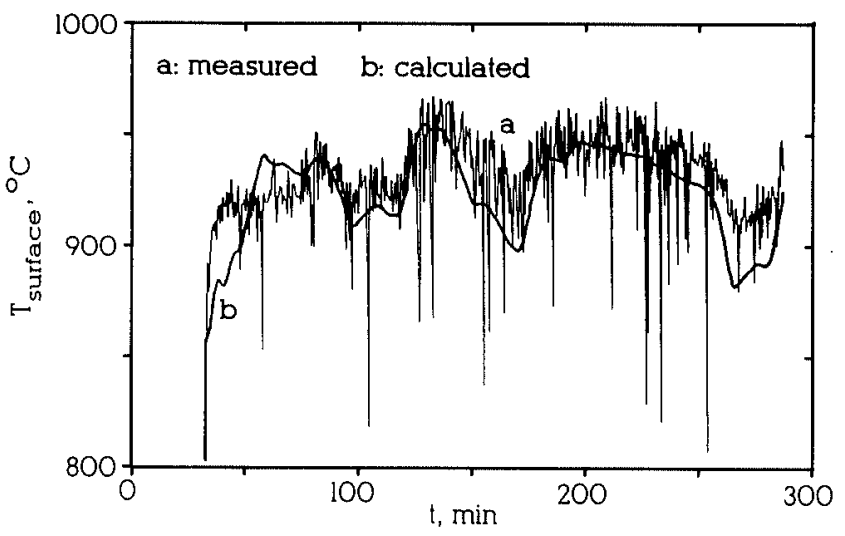

Fig. 6. Measuared and calculated surface temperature as a function of time at the end of the spray chamber (slab caster at DOFASCO Steel Works, Hamilton, Canada).

parameters yield an agreement between measurements and calculations within a wide range of casting conditions which is mostly better than $\pm 20^{\circ} \mathrm{C}$. Hence the use of an inverse model has been proven to be a valuable tool to perform such an adjustment.

An example from another installation of the model (DOFASCO Steel Works, Hamilton, Canada) is given in Fig. 6. ${ }^{25)}$ In this figure temperatures measured with a pyrometer at the exit of the spray chamber and cor- responding calculated surface temperatures are shown as a function of time.

The model is used on-line in different ways e.g. to control the crater end position and the operation of the torch cutter and it is used as a tool to improve performance of the hot direct rolling operation.

Our model is also used especially for the latter purpose at the POSCO Steel Works, Kwangyang, Korea. A report on this is given elsewhere. ${ }^{26)}$

\section{Calculation of Material Data}

To obtain reliable predictions by the mathematical model the use of the correct material data is of similar importance as the knowledge of the proper boundary conditions. Although material data have been measured for a great number of steel grades, ${ }^{21,28,29)}$ it is not always possible to find data for a grade close enough to the chemical composition of the steel to be cast. This is especially the case for steels with low contents of alloying elements where smaller variations in the composition may have a significant effect on the material data. Therefore, an algorithm has been developed to calculate the density, the thermal conductivity and the enthalpy as a function of the temperature from the chemical composition. ${ }^{27)}$ It is applicable for steel grades with sufficiently low contents of alloying elements. The model is based on the assumption of equilibrium solidification. The material data for the steel are calculated from the corresponding data for the different phases (liquid, $\delta, \gamma$, $\left.\alpha, \mathrm{Fe}_{3} \mathrm{C}\right)$.

$$
\Psi=f_{l} \Psi_{l}+f_{\delta} \Psi_{\delta}+f_{\gamma} \Psi_{\gamma}+f_{\alpha} \Psi_{\alpha}+f_{\mathrm{Fe}_{3} \mathrm{C}} \Psi_{\mathrm{Fe}_{3} \mathrm{C}}
$$

for $\Psi=\lambda, 1 / \rho, H$

The functions of $\Psi$ for the various phases have been evaluated from the available literature data and are given with equations of the form

$$
\Psi_{\mathrm{ph}}=a_{\mathrm{ph}}+b_{\mathrm{ph}} T+c_{\mathrm{ph}} T^{2}+d_{\mathrm{ph}} T^{3}+e_{\mathrm{ph}} T^{-1}
$$

In some cases different coefficients $a, b, c, d, e$ are used in different temperature ranges. ${ }^{27-29)}$ In this way in the single phase regions a good agreement could be obtained between literature data on carbon and low alloyed steels and data calculated from Eq. $(16) .{ }^{28,29)}$ The phase fractions $f_{l}, f_{\delta}, f_{\gamma}, f_{\alpha}$ and $f_{\mathrm{Fe}_{3} \mathrm{C}}$ are calculated from the phase diagram using the principle of mass conservation for each considered element.

The liquidus and solidus temperatures as well as the temperatures for the beginning and the end of the $\delta-\gamma$ transformation are taken to vary linearly with the content $[\% i]$ of the solute elements.

$$
T_{\mathrm{phc}}=T_{\mathrm{Fe}, \mathrm{phc}}+\sum_{i} Z_{\mathrm{phc}, i} \cdot[\% i]
$$

(phc: phase change, e.g. $l \rightarrow l+\delta$ )

Depending on the type of phase change $T_{\mathrm{Fe}, \mathrm{phc}}$ presents the melting point of pure $\delta$-iron $\left(1536^{\circ} \mathrm{C}\right)$, or of pure $\gamma$-iron $\left(1525^{\circ} \mathrm{C}\right)$ or the temperature of the $\delta-\gamma$ transformaton of pure iron $\left(1392^{\circ} \mathrm{C}\right)$. The coefficients $Z_{\text {phe, } i}$ were derived from the binary systems. It was checked whether they were consistent with the known 


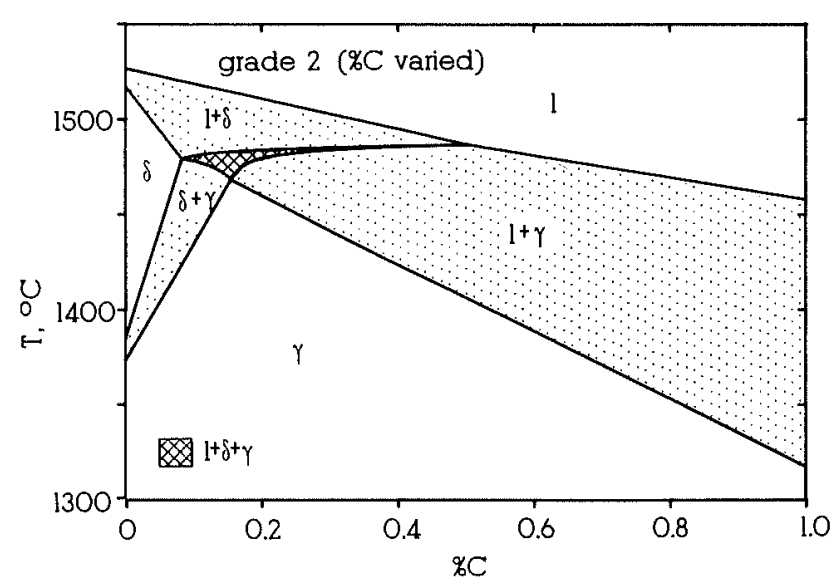

Fig. 7. Calculated $\% \mathrm{C}-T$ diagram for grade 2 (Table 2) with $\% \mathrm{C}$ varied.

Table 2. Chemical composition of two grades (1: sheet grade, 2: carbon grade)

\begin{tabular}{ccl}
\hline Elements (\%) & Grade 1 & Grade 2 \\
\hline $\mathrm{C}$ & 0.03 & 0.87 \\
$\mathrm{Mn}$ & 0.2 & 0.85 \\
$\mathrm{P}$ & 0.01 & 0.02 \\
$\mathrm{~S}$ & 0.01 & 0.005 \\
$\mathrm{Si}$ & 0.02 & 0.35 \\
$\mathrm{Cu}$ & 0.05 & 0.15 \\
$\mathrm{Ni}$ & 0.03 & 0.1 \\
$\mathrm{Cr}$ & 0.04 & 0.0 \\
$\mathrm{Mo}$ & 0.02 & 0.02 \\
$\mathrm{~V}$ & - & - \\
$\mathrm{Ti}$ & - & - \\
$\mathrm{Nb}$ & - & - \\
$\mathrm{Al}$ & 0.06 & 0.05
\end{tabular}

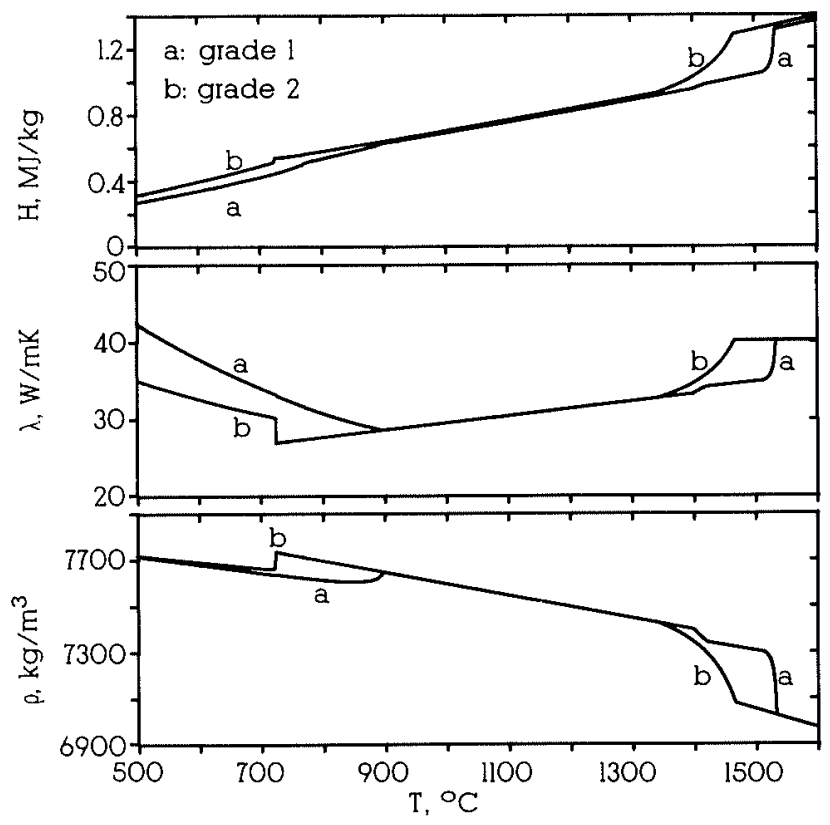

Fig. 8. Calculated enthalpy, thermal conductivity and density for grade 1 and 2 from Table 2.

heats of transformation, and if they were not they were modified so that both the phase diagram and the dilute solution law is met as closely as possible. ${ }^{27)} \mathrm{A}$ comprehensive comparison was also made on the availa- ble measured data for ternary alloys and for complex (low alloyed) steels. This part of the work will be reported in detail at a later time.

Figure 7 shows for example the \% $\mathrm{C}-T$ diagram calculated for the steel grade 2 ( $\% \mathrm{C}$ varied) for which the chemical composition is given in Table 2 . Figure 8 gives the calculated enthalpies, thermal conductivities and densities respectively as a function of temperature for both grades listed in Table 2 .

\section{Summary and Conclusion}

A mathematical model has been developed to calculate the three dimensional and instationary temperature field in the strand during continuous casting. For on-line applications the temperature field is calculated in a two-dimensional cross-sectional area in longitudinal direction in the middle of the slab.

The numerical algorithm and the computer program were tested by application to the Neumann problem for which an analytical solution is available. It was found that the analytical solution is reproduced quite closely even if relatively coarse finite difference meshes are used, which are suitable to meet computing time requirements for on-line use.

An algorithm based on the solution of the inverse heat conduction problem is applied to adjust the heat transfer coefficients for the computer model using the results of surface temperature measurements.

Another algorithm was developed to calculate the material data needed in the model from the steel composition. This algorithm is applicable to steel grades with low contents of alloying elements.

The model can be used off-line for process optimization and caster design. But the main aim of the work described in this paper was to develop a model suitable for on-line application. The model is used on-line at several casters and with adjusted boundary conditions a very good agreement between measured and calculated surface temperatures was found for the whole range of casting conditions. The results of on-line thermal tracking can be used in different ways, e.g.:

- to control the amount of spray water depending on the difference between calculated and aimed temperatures,

- to control crater end position,

- to optimize reheating times based on the calculated heat content of the slab, and

- to supply data for quality control and process optimization.

On-line quality control ${ }^{30,31)}$ is believed to become increasingly important because of the tendency to hot direct rolling. Unfortunately, it is often very difficult to relate directly process parameters to product quality. This might be easier if additional data resulting from a mathematical on-line model, e.g. surface temperature, shell thickness, temperatures at the bending point etc., are available.

\section{Nomenclature}

$$
\begin{aligned}
A_{1}, \cdots, A_{4}: & \text { coefficients in Eq. (A-1) } \\
a_{i}, b_{i}, c_{i}: & \text { empirical parameters in the formula (7) }
\end{aligned}
$$


for the heat transfer coefficient for zone $i$

$a_{\mathrm{ph}}, b_{\mathrm{ph}}, c_{\mathrm{ph}}, d_{\mathrm{ph}}, e_{\mathrm{ph}}:$ coefficients in Eq. (16)

$B_{1}, \cdots, B_{3}$ : coefficients in Eq. (A-2)

$c_{p}:$ specific heat

erf: error function, erf $x=\frac{2}{\sqrt{\pi}} \int_{0}^{x} e^{-y} d y$

$F$ : objective function to be minimized for adjustment of the parameters in the formula for $h$

$f_{l}, f_{\delta}, f_{y}, f_{\alpha}, f_{\mathrm{Fe}_{3} \mathrm{C}}:$ mass fraction of the corresponding phase

$H:$ specific enthalpy

$H_{a}(i)$ : enthalpy at grid point with current number $i$ for the previous time level

$\Delta H$ : latent heat

$h$ : heat transfer coefficient

$[\% i]$ : weight percentage of the element $i$

$m$ : number of grid points in the finite difference mesh

$n_{k}: \quad$ number of measuring positions

$n_{s}$ : number of strand slices used for the parameter optimization

$\partial / \partial n:$ derivative in the outer direction normal to the surface

$q$ : heat flux density

$T$ : temperature

$\tilde{T}$ : transformed temperature (Kirchoff transformation)

$T_{\mathrm{Fe}, \mathrm{phc}}:$ melting point of pure $\gamma$-iron or of pure $\delta$-iron or point of $\delta-\gamma$ transformation of pure iron (Eq. (17))

$\widetilde{T}(i), H(i): \quad$ temperature and enthalpy at grid point with current number $i$

$T_{l}$ : liquidus temperature

$T_{0}$ : reference temperature of $\tilde{T}$ (Eq. (2))

$T_{\mathrm{phc}}:$ temperature of a phase change

$T_{s}:$ solidus temperature

$t$ : (process) time

$\Delta t: \quad t$ increment

$v_{\text {cast }}:$ casting velocity

$x, y, z:$. coordinates, $x$ is perpendicular to the broad face and $z$ is in the casting direction

$x_{o}, y_{o}, z_{o}$ : coordinates of the boundaries

$x_{s}$ : thickness of solidified shell

$Z_{\mathrm{phc}, i}:$ coefficient in Eq. (17)

$z_{\text {top }}: \quad z$-position of the meniscus or strand top

$\Delta z: \quad z$ increment (width in the finite difference mesh)

$\varepsilon$ : emissivity

$\eta$ : parameter defined by Eq. (9)

$\kappa:=\lambda / \rho c_{p}$, temperature conductivity

$\lambda:$ thermal conductivity

$\lambda_{\text {eff }}$ : effective thermal conductivity taking account for convection

$\xi:$ process parameter in Eq. (7)

$\rho$ : density

$\sigma:$ Stefan-Boltzmann constant $(=5.67$. $10^{-8} \mathrm{~W} / \mathrm{m}^{2} \mathrm{~K}^{4}$ )

$\Psi:$ represents $\lambda, 1 / \rho$ or $H$ in Eq. (15)
Subscript

$l, \delta, \gamma, \alpha:$ liquid, $\delta$-phase, $\gamma$-phase and $\alpha$-phase

\section{REFERENCES}

1) M. D. Harshaw, C. Cochrane and R. P. Savage: Process Control System for Dofasco's No. 1 Continuous Slab Caster, presented at METEC, Düsseldorf, (1989).

2) H. Diekmann-Möller: Stahl Eisen, 107 (1987), No. 9, 433.

3) R. Brockhoff, R. Embers, C. J. Kozok and P. Monheim: Prozeßrechnerautomatisierung von Stranggießanlagen, in EDVEinsatz bei der Metallgewinnung, ed. by GDMB, VCH Verlagsgesellschaft, Weinheim, (1987), 299.

4) K. Okuno, H. Naruwa, T. Kuribayashi and T. Takamoto: Iron Steel Eng., 64 (1987), 34

5) E. A. Mizikar: Trans. TMS-AIME, 239 (1967), 1747.

6) J. K. Brimacombe: Can. Metall. Q., 15 (1976), No. 2, 163.

7) A. Perkins and W. R. Irving: Mathematical Process Models in Iron and Steelmaking, Iron Steel Inst., Amsterdam, (1973), 187.

8) B. Rogberg: Scand. J. Matall, 12 (1983), 13.

9) R. Davies, N. Blake and Ph. Campbell: Proc. 4th Int. Conf. Continuous Casting, Brussels 1988, Verlag Stahleisen, Düsseldorf, (1988), 645.

10) M. J. Mundin, J. L. R. Pimenta, C. A. G. Valadares and P. F Pereira: Proc. Continuous Casting Conf., London 1985, The Inst. Met., London, (1985), 50.1.

11) M. C. M. Cornelissen, W. Plokker and G. L. J. Bart: Proc. Solidification Processing Conf., Sheffield 1987, The Inst. Metals, London, (1988), 218.

12) K. Miyazawa and K. Schwerdtfeger: Arch. Eisenhüttenwes., 52 (1981), 415.

13) H. Jacobi and K. Schwerdtfeger: Metall. Trans. A, 7A (1976), 811.

14) J. Crank: Numerical Methods in Heat Transfer, ed. by R. W. Lewis, K. Morgan and O. C. Zienkiewicz, John Wiley \& Sons, New York, (1981), 177.

15) R. M. Furzeland: J. Inst. Maths. Applics., 26 (1980), 411.

16) T. Takawa, R. Takahashi and M. Tatsuwaki: Sumiitomo Search, 34 (1987), 79

17) S. N. Singh and K. E. Blazek: Proc. Nat. Open Hearth Basic Oxygen Steel Conf., St. Louis, Metall. Society AIME, New York, (1976), 59, 264.

18) R. Jeschar, U. Reiners and R. Scholz: 5th Int. Iron and Steel Cong., Washington 1986, Steelmaking Proc., The Iron Steel Soc. AIME, Warrendale, Penn., (1986), 511.

19) J. K. Brimacombe, I. V. Samarasekera and J. E. Lait: Continuous Casting, Vol. II, The Iron Steel Soc. AIME, Warrendale, Penn., (1984).

20) K. Schwerdtfeger: Erstarrung metallischer Schmelzen, ed. by P. R. Sahm, DGM-Verlag, Frankfurt, (1988), 205.

21) F. Richter: Die wichtigsten physikalischen Eigenschaften von 52 Eisenwerkstoffen, Verlag Stahleisen, Düsseldorf, (1973).

22) K.-H. Tacke: Int. J. Numerical Methods Eng., 21 (1985), 543.

23) M. Larrecq, J. P. Birat, C. Saguez and J. Henry: Proc. 3rd Process Technology Conf., Pittsburgh 1982, The Iron Steel Soc. AIME, Warrendale, Penn., (1982), 273.

24) L. Holappa, E. Laitinen, S. Louhenkilpi and P. Neittaanmäki: Proc. 24th Annual Conf. of Matallurgists, Vancouver 1985, CIM, Montreal, (1985), 242.

25) M. Harshaw: Thermal Tracking, Internal Report, DOFASCO Inc., (1988).

26) J. S. Kim, U. S. Yoon, B. J. Kim, K. CH. Shin and J. Y. Chung: ATS Steel Conference, Paris, December, ATS, Paris, (1991).

27) K. Schwerdtfeger and K. Harste: Calculation of Material Data from the Chemical Composition, Internal Report, Inst. für Allgemeine Metallurgie, Technical University Clausthal, (1988).

28) K. Harste: Untersuchung zur Schrumpfung und zur Entstehung von mechanischen Spannungen während der Erstarrung und nachfolgenden Abkühlung zylindrischer Blöcke aus $\mathrm{Fe}-\mathrm{C}$ Legierunger, Thesis, Technical Unsiversity Clausthal, (1989).

29) A. Jablonka, K. Harste and K. Schwerdtfeger: Steel Res., 62 (1991), No. 1, 24.

30) W. R. Irving: Proc. 4th Int. Conf. Continuous Casting, Brussels 1988, Verlag Stahleisen, Düsseldorf, (1988), 25. 
31) T. Fastner, A. Mayr, P. Narzt and F. Wallner: Proc. 4th Int. Conf. Continuous Casting, Brussels 1988, Verlag Stahleisen, Düsseldorf, (1988), 142.

\section{Appendix}

For simplicity the numerical algorithm is outlined for the one-dimensional Eq. (1-b) with $v_{\text {cast }}=0$. The extension to the general Eq. (1-a) is straightforward. Using finite differences and an implicit scheme in time, discretization of Eq. (1-b) gives a system of equations each having the form

$$
\begin{array}{r}
A_{1}(i) \tilde{T}(i-1)+A_{2}(i) \tilde{T}(i)+A_{3}(i) \tilde{T}(i+1) \\
\quad+A_{4}(i) H(i)=H_{a}(i) \quad \ldots \ldots \ldots \ldots \ldots \ldots \ldots
\end{array}
$$

where $i$ is the current number of an internal grid point. $H_{a}(i)$ is the known specific enthalpy at grid point $i$ for the previous time level. To obtain an equation for the variable $H$ solely, Eq. (A-1) can be rewritten to give

$$
B_{1}(i) H(i-1)+B_{2}(i) H(i)+B_{3}(i) H(i+1)=H_{a}(i)
$$

with

$$
\begin{aligned}
& B_{1}(i)=A_{1}(i) \cdot \frac{\tilde{T}(i-1)}{H(i-1)} \\
& B_{2}(i)=A_{2}(i) \cdot \frac{\tilde{T}(i)}{H(i)}+A_{4}(i) \\
& B_{3}(i)=A_{3}(i) \cdot \frac{\tilde{T}(i+1)}{H(i+1)}
\end{aligned}
$$

Two equations for the grid points on the boundary are derived from the boundary conditions and can be brought to the forms (A-4a) and (A-4b). The complete system of equations which is solved iteratively is then

$$
\begin{aligned}
& \tilde{T}(1) \quad+C_{1}(1) \quad H(2) \quad=C_{2}(1)
\end{aligned}
$$

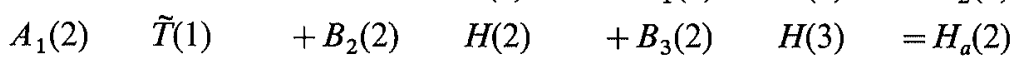

$$
\begin{aligned}
& B_{1}(i) \quad H(i-1)+B_{2}(i) \quad H(i) \quad+B_{3}(i) \quad H(i+1)=H_{a}(i) \quad 3 \leq i \leq m-2 \\
& B_{1}(m-1) H(m-2)+B_{2}(m-1) H(m-1)+A_{3}(m-1) \tilde{T}(m) \quad=H_{a}(m-1) \\
& C_{1}(m) \quad H(m-1)+\quad \tilde{T}(m) \\
& =C_{2}(m)
\end{aligned}
$$

For the first iteration cycle $\widetilde{T}$ from the previous time level is taken to calculate the material data $\lambda, \rho$ entering $A_{1}, \cdots, A_{4}$ and $C_{1}, C_{2}$. The ratio $\tilde{T} / H$ at each internal grid point is also taken from the previous time level. This enables to calculate $B_{1}, B_{2}, B_{3}$ and $C_{1}$ and, therefore, to solve the linearized system of equations yielding new enthalpies $H$ at internal and new temperatures $\widetilde{T}$ at boundary grid points. Using the known $H-\widetilde{T}$-relationship new temperatures, material data, values for the $\tilde{T} / H$-ratio and, therefore, new values for $A_{1}, \cdots, A_{4}, B_{1}, B_{2}, B_{3}$, $C_{1}, C_{2}$ are obtained and the system of equations is again solved. This procedure is repeated until a prescribed stability for $H$ and $\widetilde{T}$ is reached. Then the temperatures for the next time level are calculated in the same way.

The material data relations $\tilde{T}(T), T(\widetilde{T}), \widetilde{T}(H) H(\widetilde{T})$, $\lambda(\tilde{T}), \rho(\widetilde{T})$ which are needed during the calculation are established in the computer program in the form of an integer-indexed vector. The easiest way to do this is to set for instance for the integer $i=1000 \lambda(i)=\lambda(T=$ $1000^{\circ} \mathrm{C}$ ). How to obtain a finer discretization is straightforward. The components of these vectors are evaluated only once at the start of the computer program. In this way the material data can be calculated during the iteration by only few operations (one interpolation) even if complicated functions are involved in the material data relations. 